\title{
Construcción de paz en escuelas rurales desde la perspectiva filosofía e infancia: discursos y prácticas
}

\author{
Paola Andrea Lara Buitrago, ${ }^{1}{ }^{\circledR}$, Óscar Pulido Cortés ${ }^{(1)}$ \\ Universidad de Boyacá, Universidad Pedagógica y Tecnológica de Colombia, Colombia
}

Autor de correspondencia: ${ }^{1}$ paola.lara@uptc.edu.co Recibido: 13 de julio de 2021 Revisado: 04 de agosto de 2021 Aprobado: 21 de octubre de 2021 Publicado: 07 de diciembre de 2021

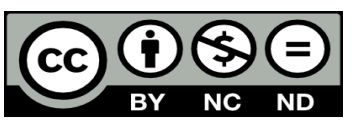

\section{Resumen}

El artículo presenta los resultados del proyecto de investigación Formación de maestros para la promoción de cultura de paz: una perspectiva ética y política, el cual analiza los discursos y las prácticas de profesores de escuelas rurales para la construcción de paz y la generación de ambientes de convivencia y de espacios pacíficos. La investigación asume la perspectiva cualitativa desde la teoría fundamentada. Se realizaron entrevistas como instrumento de recolección de información y se usó el software Atlas.ti en tanto herramienta de análisis. Las categorías emergentes del análisis fueron: contexto, significados en torno de la construcción de paz, prácticas filosóficas en la infancia, acciones y proyecciones, las cuales permitieron discusiones y el hallazgo de prácticas para la convivencia y ambientes de paz en perspectiva filosófica. La sistematización de los resultados mostró que la convivencia y la resolución de conflictos como espacios para construir paz se optimizan a partir del desarrollo del programa Filosofía para Niños y de la perspectiva filosofía e infancia, apoyadas en el cuidado, el diálogo y el cuestionamiento para la configuración de sujetos y prácticas pedagógicas en las escuelas rurales.

Palabras clave: formación de profesores, ético-política, paz, enseñanza de la filosofía

Para citar este artículo: Lara, P., \& Pulido-Cortés, O. (2021). Construcción de paz en escuelas rurales desde la perspectiva filosofía e infancia: discursos y prácticas. Praxis \& Saber, 12(31), e13145. https://doi.org/10.19053/22160159.v12.n31.2021.13145 


\title{
Peacebuilding in rural schools from the Philosophy and Childhood perspective: discourses and practices
}

\begin{abstract}
The article presents the results of the research project titled Teacher training for the promotion of a culture of peace: an ethical and political perspective, which analyzes the discourses and practices of rural schoolteachers for peacebuilding and for the creation of coexistence environments and peaceful spaces. The research adopts a qualitative perspective from the grounded theory. Interviews were conducted as a data collection instrument and the Atlas.ti software was used as an analysis tool. The emerging categories from the analysis were: context, meanings on peacebuilding, philosophical practices in childhood, actions, and projections, which allowed for discussions and for the discovery of practices for coexistence and peace environments from a philosophical perspective. The systematization of the results showed that coexistence and conflict resolution as spaces for building peace are optimized through the development of the Philosophy for Children program and of the Philosophy and Childhood perspective, based on care, dialogue, and questioning to configure individuals and pedagogical practices in rural schools.
\end{abstract}

Keywords: teacher education, ethic and politic, peace, philosophy teaching

\section{Construção da paz nas escolas rurais a partir da perspectiva filosofia e infância: discursos e práticas}

\section{Resumo}

O artigo apresenta os resultados do projeto de pesquisa Formação de professores para a promoção de uma cultura de paz: uma perspectiva ética e política, que analisa os discursos e práticas dos professores nas escolas rurais para a construção da paz e a geração de ambientes de convivência e espaços pacíficos. A pesquisa adota uma perspectiva qualitativa baseada na teoria fundamentada. As entrevistas foram realizadas como um instrumento de coleta de dados e o software Atlas.ti foi usado como ferramenta de análise. As categorias que emergiram da análise foram: contexto, significados sobre a construção da paz, práticas filosóficas na infância, ações e projeções, as quais permitiram discussões e a descoberta de práticas para a convivência e ambientes de paz numa perspectiva filosófica. A sistematização dos resultados mostrou que a convivência e a resolução de conflitos como espaços para a construção da paz são otimizadas através do desenvolvimento do programa Filosofia para Crianças e da perspectiva filosofia e infância, baseadas no cuidado, no diálogo e no questionamento para a configuração de sujeitos e práticas pedagógicas nas escolas rurais.

Palavras-chave: formação de professores, ética-política, paz, ensino de filosofia 
${ }^{1}$ La paz es una exigencia para Colombia y es necesario pasar del discurso teórico, político y jurídico a materializarla en prácticas concretas donde los sujetos involucrados asuman posturas y acciones que transformen comunidades e impacten en los procesos de subjetivación. En esto radica la importancia de los procesos educativos que tengan como foco la educación para la paz, que según la Unesco (2000, como se citó en Ministerio de Educación de Colombia [MEN], 2015) es el

proceso de promoción de conocimientos, habilidades, actitudes y valores necesarios para lograr cambios de comportamiento que permitan a niños y niñas, jóvenes y adultos prevenir los conflictos y la violencia, tanto manifiestos como estructurales, resolver los conflictos pacíficamente y crear las condiciones propicias para la paz, ya sea en un nivel intrapersonal, interpersonal, intergrupal, nacional o internacional. (p. 6)

En Colombia, luego de más de cincuenta años de conflicto armado y de la firma de acuerdos para la paz, el Congreso de Colombia y la Presidencia de la República expidieron respectivamente la Ley 1732 de 2014 y el Decreto 1038 de 2015, para establecer nuevos horizontes en torno a la formación para la paz. Un ejemplo es la Cátedra de la Paz, la cual no solo es una asignatura, sino que busca convertirse en un ambiente de reconciliación, de convivencia y de acciones de paz (art. 2, Decreto 1038, 2015). De igual forma, el Acuerdo final para la terminación del conflicto y la construcción de una paz estable y duradera (Gobierno Nacional \& FARC-EP, 2016) asume el sector rural y sus procesos educativos y formativos como ejes centrales para el logro de los objetivos propuestos, donde aparecen los siguientes focos de desarrollo: salud, educación y vivienda.

El Plan especial de educación rural hacia el desarrollo rural y la construcción de paz (MEN, 2017) considera la educación rural como parte fundamental de la formación en, desde y para el posconflicto, que implica la búsqueda de estrategias pedagógicas por parte de los maestros en la enseñanza y en la promoción de ambientes de paz (Martínez-Restrepo et al., 2015; Misión para la Transformación del Campo, 2015), con el propósito de generar y enfocarse en distintas acciones que favorezcan la interacción de los estudiantes en escenarios ético-políticos propios para el respeto y la convivencia (Arboleda et al., 2017). La escuela rural es una estrategia clave en la formación de sujetos y, a su vez, en la vinculación con las comunidades. También es el centro de articulación de proyectos productivos y un lugar de encuentro y de reconciliación (MEN, 2015). Además de recoger inquietudes y sueños de quienes conviven alrededor, la escuela rural es el lugar donde se construyen experiencias y expectativas que promueven posibilidades de acción y de transformación en la población infantil, profesores y familias. Por eso, el planteamiento de experiencias pedagógicas entorno a la paz impacta en las familias, en el contexto y en los maestros, quienes son promotores y formadores de paz, de convivencia y de reconciliación. Sus acciones se relacionan con la Cátedra de la Paz e inciden en la comunidad. Asimismo, los maestros son agentes importantes dentro de la sociedad, pues son sujetos que se empoderan, fortalecen la convivencia y generan un discurso ético y político en su entorno.

En esta necesidad de generar espacios de formación ético-política en la escuela rural, el programa de Filosofía para Niños (FpN) tiene como objetivo la formación ética y política

1 Artículo de investigación del proyecto Formación de maestros para la promoción de la cultura de paz: una perspectiva ética y política. COLCIENCIAS-UPTC SGI 2620, convocatoria No. 812 de 2018, actividad de Ciencia, tecnología e Innovación: innovación social y el eje Promoción de la cultura de paz y reconciliación. 
desde la infancia mediante el fortalecimiento del ejercicio del pensar (Unesco, 2007). Se le considera potente para la generación y la construcción de espacios de convivencia y de prácticas democráticas en torno al cuidado y a la preocupación por los sujetos y por los otros. FpN propicia la formación de habilidades del pensamiento, el diálogo, el razonamiento y la democracia. También ha sido referente teórico y práctico para diferentes instituciones educativas de Colombia que han proyectado cambios e innovaciones en las prácticas pedagógicas.

FpN permite pensar juntos, reflexionar sobre la realidad, reconocer la potencia del pensamiento, crecer con el otro y mirar en retrospectiva el ejercicio del maestro y de la escuela como ámbito de reflexión, de criticidad, de diálogo, de juicio y de razonamiento. De igual manera, ubica la escritura y la lectura como experiencias de pensamiento y de creación. FpN es el pretexto para pensar y transformar las prácticas escolares en relación con el pensamiento y con la pregunta como detonante. Así, cada pregunta, cada diálogo, cada argumento y cada creación comprometen actitudes de cuidado de sí mismo, del otro y de lo otro, que se consideran apropiadas para las interacciones entre pares, para relaciones entre la escuela y la comunidad y para el conocimiento del entorno. Por ello, para construir ambientes de paz, se necesita configurar prácticas de reconocimiento, ubicarse en el lugar del otro, razonar sobre situaciones, decidir sobre los actos y reflexionar a partir de elecciones.

Las experiencias de FpN sistematizan varias posibilidades para transformar la escuela desde la convivencia y el pensamiento. En efecto, la preocupación por la formación de maestros moviliza la lectura de las prácticas escolares para posibilitar la resignificación de la paz en la escuela en escenarios, discursos y prácticas, con el fin de aportar a la consolidación de saberes y de experiencias que justifiquen la terminación del conflicto en las instituciones educativas para construir un mejor país. En efecto, la muestra seleccionada corresponde a escuelas rurales del departamento de Boyacá que han incluido en sus currículos la perspectiva filosofía e infancia ${ }^{2}$ (PFI) para plantear soluciones a problemáticas próximas a las niñas, niños y jóvenes, e incluso a la comunidad. Así, en la dinámica de trabajo, se construyen discursos y prácticas.

El artículo se estructura en cuatro partes: el fundamento teórico, la metodología, los resultados - organizados según las categorías: contextos, significados alrededor de la construcción de paz, prácticas filosóficas en la infancia, acciones y proyecciones para construir paz en la escuela - y conclusiones.

\section{Sobre la formación ético-política}

Los fundamentos y las razones por las que un programa como FpN apoya la formación ética y política de niñas, niños y jóvenes son la posibilidad de reflexionar sobre la acción y de justificar los criterios por los cuales se toman decisiones o se enfrentan a situaciones de la cotidianidad. De este modo, la construcción de paz y la consolidación de ambientes de convivencia visibilizan situaciones y problemas que requieren el razonamiento, la reflexión y la indagación para argumentar sobre las mejores maneras de solucionar conflictos. Por ello, la formación en el diálogo, en la pregunta y en la investigación moviliza el cuestionamiento por la actuación del sujeto y las posibilidades para determinar criterios y fundamentos

2 La PFI es una línea de investigación que se dedica a liderar, apoyar y plantear espacios de intercambio académico y al fortalecimiento de las prácticas educativas en torno al programa FpN. 
basados en el lenguaje, en las emociones y en el cuidado, para una formación individual.

FpN plantea la formación ética como la identificación y la comprensión de las creencias, de las preguntas y de los supuestos morales. Esta formación se fundamenta en dos momentos: educación moral e investigación ética. La educación moral se refiere a la formación de los hábitos. La investigación ética consiste en examinar aquellos factores que determinan una situación ética, es decir, las circunstancias de la acción, los motivos, las consecuencias, el lenguaje, los sentimientos y las emociones (Pineda, 2004). Según Lipman (1988) la investigación ética prepara a los niños para una práctica moral razonada y necesita formar habilidades de razonamiento, de investigación y de formación de conceptos, estados mentales, actos mentales y actos metacognitivos. Estas herramientas constituyen la formación en ética aplicada en situaciones diversas, y no planteadas únicamente desde los dilemas éticos o los conceptos.

Las comunidades de indagación son el espacio para la formación ética en la medida que se reflexionen problemas y posibles alternativas de actuación, al pasar por el ejercicio de pensamiento y por la construcción de criterios y juicios. La multiplicidad de perspectivas favorece el pensamiento multifuncional (Lipman, 2016), donde la criticidad, la creatividad y el cuidado permiten entretejer sentidos y construcciones de realidad. La comprensión de saberes y la consolidación de conocimientos son fundamentos para actuar y decidir sobre el estar en el mundo, observados desde la dimensión cognitiva. A pesar de que se conozca la importancia de pensar crítica y creativamente, el cuidado conserva un lugar de totalidad y de completitud, puesto que existe la preocupación por otro que habita y por un entorno habitable, lo cual demuestra compromiso por el lugar donde se vive y se interactúa.

La construcción de paz en términos del cuidado es una opción para activar la preocupación, el conocimiento y la comprensión del sujeto, que en las aulas son los compañeros, los amigos o los profesores. También es identificar que la existencia del otro permite deconstruir y reconstruir argumentos, puesto que la emisión de juicios implica que se examinen las razones por las cuales se da respuesta a un acontecimiento. Estas razones son enseñadas a otros, quienes, en la misma posición de sujetos de diálogo, propician el intercambio, la pregunta y la discusión para escuchar, criticar y revisar lo planteado. Así, el carácter de cuidado se recrea en el narrarse, en el preguntarse, en el leerse y en el escribirse con el otro (Larrosa, 1995), según las subjetividades y la coconstrucción de sentires y de pensares.

En FpN se identifica cómo la fundamentación del programa sostiene que la ética y la política son un asunto que compete a todos con un carácter de formación; y que la escuela es el escenario para que se cultive el deseo de saber, de conocer, de aprender y de convivir. En efecto, construir paz y propiciar ambientes de paz en la escuela abarcan las interacciones con el otro movilizadas por el ejercicio de pensar y el cuidado.

\section{Metodología}

La investigación se enmarca en la perspectiva cualitativa porque la búsqueda de la comprensión de la realidad educativa se genera a partir de interpretaciones y del descubrimiento de procesos donde participan actores, en particular, los profesores de escuelas rurales en el departamento de Boyacá, Colombia, que desarrollan sus acciones de 
construcción de paz en la PFI.

Sobre esta mirada, la teoría fundamentada "es una metodología de análisis, unida a la recogida de datos, que utiliza un conjunto de métodos, sistemáticamente aplicados, para generar una teoría inductiva sobre un área sustantiva" (Glaser, 1992, como se citó en Torre, 2013, p. 4). La comprensión intersubjetiva de las percepciones de maestros a través de los discursos y de las prácticas se recolectó mediante entrevistas semiestructuradas, que permitieron categorizar las experiencias pedagógicas en contextos rurales y las proyecciones para la construcción de paz.

La información se codificó a través de la herramienta Atlas Ti. La codificación de datos se hizo con codificación abierta. Se relacionaron los términos como códigos determinantes de la información recogida: resolución de conflictos, construcción de paz y FpN. Luego, la codificación axial interconectó códigos con las categorías: contextos, significados alrededor de la construcción de paz, prácticas filosóficas en la infancia, acciones y proyecciones para construir paz, apoyadas en condiciones y contextos. Por último, en la codificación selectiva, se seleccionó la categoría central y su relación con las otras categorías emergentes.

La codificación con Atlas.Ti permitió organizar la información en documentos primarios, códigos, citas, memos y redes, lo cual facilitó ordenar y auscultar las relaciones y condiciones que se entretejen en la información recogida. Con base en la fundamentación de códigos y en su densidad, se señalaron los resultados y se dispusieron en las categorías mencionadas arriba.

\section{Resultados}

El análisis de los discursos y de las prácticas para la educación de la paz se relaciona con el conocimiento del contexto, con los significados alrededor de la construcción de paz y con las relaciones en las dimensiones ética y política, en las prácticas filosóficas en la infancia $y$ en las acciones y proyecciones para construir paz en la escuela y generar un cambio en el país.

\section{Contextos}

La pregunta por el contexto de las escuelas rurales consolida percepciones y apreciaciones de situaciones que se enfocan en acciones y prácticas pedagógicas para gestar discusiones entorno a problemáticas observadas o vividas. Estas conversaciones se organizan respecto a la ruralidad, al conflicto armado y a las condiciones escolares, las cuales muestran cómo la PFI introduce y moviliza experiencias alrededor de la paz.

Ruralidad. En Colombia, el 21 \% del total de la población del país - mestiza, indígena, afrocolombiana, entre otras- habita en zonas rurales (Trivelli \& Berdegué, 2019). Así mismo, el desarrollo productivo de Boyacá reposa principalmente sobre las actividades agrícolas y ganaderas. Su ubicación en la Región Andina privilegia la obtención de lácteos, papa, cacao, hortalizas, frutales, caña y panela, entre otros (Agencia de Desarrollo Rural, 2019). Así mismo, el sector agropecuario cuenta con organizaciones y federaciones que vigilan, respaldan y administran el territorio desde la normativa y los lineamientos para la comercialización y mercado de los pequeños productores. El departamento tiene debilidades como la desnutrición, la inseguridad alimentaria, la baja formación de jóvenes para el acceso 
a la educación superior, los procesos migratorios campo-ciudad y el envejecimiento de la población, sumados a los altos niveles de pobreza y de desigualdad entre las áreas urbanas y rurales. La pobreza multimodal en la ruralidad representa el $75 \%$, mientras que en el área urbana es de 38 \% (Agencia de Desarrollo Rural, 2019). La educación en la ruralidad enfrenta desigualdades. La brecha entre los ámbitos educativos rural y urbano se abre cada vez más debido a problemas como: la deserción escolar a causa de desplazamientos, las largas distancias hacia los centros educativos, la presencia de población flotante - residencia temporal-, la necesidad de trabajar a cambio de la formación educativa, la precariedad de condiciones mínimas de sostenibilidad y la falta de servicios públicos y de conectividad. Solo el 7,2 \% de la población del departamento tiene acceso a internet. La cobertura en educación media representa el 48,4 \% y en secundaria el 74,9 \% (Agencia de Desarrollo Rural, 2019).

Además de las cifras enunciadas, los docentes entrevistados describen la educación rural con problemas de financiación, de interacción y de proyección social: "existe ausencia de servicios públicos básicos, dificultades con las estructuras físicas, los salones y campos deportivos. Además, la tecnología no está a disposición de los estudiantes" (Entrevistado [E] 5).

Las escuelas rurales identifican familias que priorizan la educación de los niños y están en disposición de apoyarlos en sus procesos de aprendizaje. En contraste, otras comunidades les dan poca importancia a la institucionalización del saber y a la continuación de la escolarización, pues expresan lo innecesaria e injustificada que es la formación personal: "la aspiración es a tener dinero. No es realmente que los estudiantes hagan transformación social" (E2). Factores como la carencia económica inciden en esta visión.

Estas razones se reflejan en el seguimiento, en la preocupación y en la respuesta de padres de familia o acudientes para participar en las actividades de las escuelas. Las actividades económicas de la región se centran en la agricultura, en el sector minero y en la construcción. Al interior de las familias, en ocasiones, los estudiantes apoyan el desarrollo de las actividades económicas, "ayudando a sus papás en las labores del campo o trabajos afines al sustento" (E2). La mayoría de los estudiantes que asisten a las escuelas rurales pertenecen a familias con bajos niveles de calidad de vida. También hacen parte de familias numerosas, las cuales son en su mayoría campesinas. Además, en la ruralidad existe desplazamiento en el territorio por necesidad de reubicación a causa del desempleo, lo cual produce la migración hacia ciudades con mayor actividad laboral. De igual forma, una problemática que caracteriza al departamento es el alto consumo de alcohol y los problemas de violencia intrafamiliar.

Conflicto armado. Boyacá tiene antecedentes de conflicto armado de grupos al margen de la ley. Por su ubicación geográfica, tiene zonas fronterizas con otros territorios que influyen en hechos de violencia como acciones bélicas, secuestros, desplazamientos, desapariciones, homicidios y muertes de civiles en conflicto (Hernández, s. f.). Las 45121 víctimas de conflicto armado (Unidad para la Atención y Reparación Integral a las Víctimas, 2020) fueron afectadas en hechos relacionados con la territorialidad, con atentados a la integridad personal y con la inseguridad. Según información del Observatorio de Memoria y Conflicto y del Centro de Memoria Histórica (2019), las cifras de los casos de violencia en 
el periodo 1959-2016 responden a: 627 acciones bélicas, 61 masacres, 921 desapariciones, 2 atentados terroristas y 23 ataques a poblaciones, entre otros hechos.

Una de las experiencias estudiadas se ubica en el conflicto por el paramilitarismo, el cual se "sufrió durante la época desde el 2002, una violencia bastante radical, y he tenido bastantes estudiantes quienes narran desde sus vivencias cómo fue este conflicto y cómo ellos lo vivenciaron" (E6). Estos antecedentes han transformado el pensamiento de los estudiantes para evitar que vuelvan a ocurrir épocas de violencia. Por eso, en ocasiones se vuelve sobre los hechos para la no repetición e incluso se va a sitios donde se realizaron hechos violentos: "este lugar fue un lugar específico de violencia marcada en donde muchos de los estudiantes fueron a hacer un reconocimiento de esa zona donde muchos familiares de ellos después los desaparecieron" (E6). Estas narrativas fueron un insumo importante para trabajar en la perspectiva de FpN.

Condiciones escolares. Las escuelas rurales presentan escenarios pertinentes paragenerar diálogos en ambientes de aprendizaje abiertos: bohíos, ágoras, granjas. El "acercamiento con la naturaleza es un ambiente apropiado para pensar y reflexionar sobre nuestro entorno" (E6). Estas condiciones exaltan el reconocimiento del territorio y la apropiación de saberes autóctonos y tradicionales del pensamiento rural y campesino, construidos desde culturas indígenas como los muiscas. Esta riqueza intelectual de los mayores, los estilos de vida, las relaciones entre la comunidad, las creencias, el ambiente y los objetos que constituyen la cultura promueven conocer las prácticas éticas y de cuidado. La preocupación por la dimensión ecológica conduce a la comprensión de las dinámicas ambientales, al cuidado de la tierra y a la protección ambiental en los proyectos educativos.

Como resultado de la sensibilidad por el contexto, las comunidades de indagación operan alrededor de los principios de diálogo, de escucha y de cuestionamiento, lo cual posibilita que los compromisos éticos, de responsabilidad social y el liderazgo de comunidades impacten en las actitudes y en las transformaciones con la participación de los niños. Las acciones en la PFI han transformado actitudes y lo que antes eran lugares y momentos de pasividad de la voz de los sujetos. Las experiencias filosóficas han contribuido a la acción de hablar por sí mismos, de presentar sus posiciones y de expresar pensamientos: "en la forma en que hablan con determinados temas ya no son tan callados y son mucho más participativos" (E1). En conclusión, el contexto justifica acciones y genera condiciones para repensar las prácticas de la escuela rural y las transformaciones a nivel personal, comunitario, educativo y pedagógico desde la perspectiva filosófica.

\section{Significados alrededor de la construcción de paz}

La Ley 1732 (Congreso de Colombia, 2014) y el Decreto 1038 (Presidencia de la República, 2015) presentan la Cátedra de la Paz. Este último enfatiza en la necesidad de una educación para la paz como "la apropiación de conocimientos y competencias ciudadanas para la convivencia pacífica, la participación democrática, la construcción de equidad, el respeto por la pluralidad, los Derechos Humanos y el Derecho Internacional Humanitario". La Cátedra de la Paz en Colombia se conceptualiza a partir de la convivencia pacífica, de una educación equitativa, incluyente y democrática, y de la formación ciudadana. Se fundamenta principalmente en la resolución de conflictos y en el reconocimiento de la diversidad y del multiculturalismo como factores que caracterizan e intervienen en las relaciones de respeto 
y de no violencia. La Cátedra de la Paz "responde al desafío de formar seres humanos con capacidad para el cuidado de sí mismo, de los demás y del medio ambiente" (MEN, 2015, p. 5), reflejada en las categorías: convivencia pacífica, participación ciudadana, diversidad e identidad, memoria histórica y reconciliación, desarrollo sostenible y ética, cuidado y decisiones. Al considerar este marco normativo, se agrupan los discursos de los maestros en relación con la paz, identificados en: percepciones sobre la Cátedra de la Paz, significados en torno a la paz, la paz en relación con la ética y la política.

Percepciones sobre la Cátedra de la Paz. Algunos profesores describen que la paz no depende de un decreto y que la Cátedra de Paz no soluciona la discusión ni mejora la convivencia en un horario específico ni en un espacio en el currículo. Por el contrario, la paz tiene que salir del aula y ser una preocupación común y una obra conjunta, es decir, que todos aporten a su construcción en todo momento, en cada actividad que se realiza dentro y fuera de la escuela: "todo va inmerso en el diario vivir" (E5). La materialización de la Cátedra de la Paz se relaciona con experiencias pedagógicas que se realizan en torno al diálogo y al ejercicio democrático: "espacios de diálogo con los niños en los que se toman algunos recursos como pueden ser lecturas, cortos o también situaciones de la vida cotidiana que viven los niños. En esas actividades se busca sobre todo problematizar" (E3). Así, adquieren sentido situaciones que previamente son estudiadas para justificar maneras de actuación razonadas. La construcción de acuerdos para mediar en conflictos, apoyados en las razones previamente analizadas, son acciones para promover la democracia, la ciudadanía y la convivencia (Rivas, 2017). Sin embargo, antes del decreto de la Cátedra de la Paz, algunas acciones desarrolladas a nivel institucional consolidaron aportes a la construcción de paz, por ejemplo: semanas de la conciencia ciudadana; campañas de cuidado con énfasis en el afecto, en la amistad y en el manejo de la agresividad en conflictos; propuestas de abrazoterapia; vacunas contra la agresividad; padrinazgo; saludo ciudadano; entre otras.

Estas prácticas posibilitan que las nociones de cuidado se asuman desde las escuelas rurales para la construcción de la paz. Por ejemplo, el producto de las amistades forja una ética con las interpelaciones subjetivas (Pagni, 2016), una ética con elementos propios, los cuales recrean percepciones de habitar en paz. Por ello, la modificación en los modos de existencia tendientes a la consolidación de una cultura de paz resiste y conserva más condiciones que propician el acercamiento y la confianza con el otro.

Un aspecto que relaciona la Cátedra de la Paz con el sentido de la singularidad -o el reconocimiento de la subjetividad- es la decisión de actuar. Las dinámicas en relación con la Cátedra de la Paz se ubican en el buen actuar desde uno mismo y en el apoyo de organizaciones no gubernamentales que gestionan trabajo sobre la ciudadanía y la democracia, con la adquisición y la promoción de formas para la discusión y recursos que apoyan la cátedra, tales como literatura, materiales lúdicos, campañas y proyectos.

Significados en torno a la paz. En términos de cuidado, la paz se propone como una condición para vivir en armonía con el sujeto, con los otros y con el entorno, donde se comparten las experiencias de vida. La relación de cuidado afecta el reconocimiento y la comprensión en comunidad e implica inicialmente un descubrimiento como ser individual, que después toma sentido en la convivencia con otros. Así, la paz se considera como "construcción permanente individual, inicialmente, del respeto a uno mismo. En ese 
sentido, entonces, ya lo que trasciende con los otros y lo que podríamos hablar de una convivencia es vivir con el otro" (E4). La comunidad, en sus dinámicas de interrelación, invita a que una construcción desde la individualidad afecte, modifique y opere en los demás: "es un ejercicio que se realiza en colectivo, ya que desde la individualidad no se construye comunidad y tampoco se podría construir la paz" (E3), de modo que la paz, en términos de fundamentar una estética de la existencia, ubica la dimensión de conciencia, de reflexión y de estado armónico. Así, es un "estado de tranquilidad y de buen vivir. Relaciono así porque, dentro del proyecto de FpN, nosotros hacemos énfasis en el principio filosófico de Foucault del cuidado de sí, el cuidado del otro y de lo otro" (E6).

La paz implica los derechos humanos y la ciudadanía en la medida que promueve la participación y la democracia. Además, permite tener conciencia de los sujetos y convivir en términos de preocupación por el otro, en medio de actuaciones razonadas y precaución en la no agresión: "la conciencia que tiene un sujeto sobre las dimensiones de sus decisiones sobre el alcance de sus decisiones" (E7). El reconocimiento y la existencia del otro ubica ética y políticamente la constitución de los sujetos de escuela. Existe una responsabilidad y un pensamiento cuidadoso donde las palabras y las acciones generan efectos en los demás.

La paz en relación con la ética y la política. Desligar la ética de la política no es asunto fácil, pues la ética deviene política en la construcción de paz. Ambos elementos acontecen en el encuentro del sujeto, es decir, "es de acción, de cuidado, de que nosotros entendamos que cada acto ético es el respetar al ser humano y a todo su entorno, y a los que no son humanos también" (E2). La ética lleva a entender y a tratar la sensibilidad por lo que está alrededor. Surge de uno mismo con impacto en la otredad. El cuidado atraviesa la formación ética y política. Esta noción representa la síntesis de lo que la PFI define como pensamiento cuidadoso: "una persona da cuenta de sí misma en la medida en que reconoce la existencia de otro" (E7). Es ser consciente del trato y del reconocimiento de lo que constituye todo escenario, es decir, la noción del cuidado se refleja en el territorio, en la comunidad, en la escuela y en las personas con las que conviven niños y niñas.

La paz en relación con la ética y la política fortalece el vínculo con el pensamiento, "pues el proyecto de $\mathrm{FpN}$ nos ha dado una base importantísima en la transformación del pensamiento" (E5). Esto implica su desarrollo a partir de la construcción y de la fundamentación de las razones, es decir, justificar elecciones o decisiones que llevan al sujeto a actuar, a hablar autónomamente, a deconstruir lo correcto y a trasladar las razones a diferentes ámbitos y escenarios. Por último, la definición de paz se conecta con la ética y la política para construirse como término y materializarse en acciones concretas con los estudiantes y con la comunidad de las escuelas rurales. Así mismo, la formación ética y política es un fundamento del programa de $\mathrm{FpN}$ y se considera insumo para recrear la invención de la escuela contemporánea.

\section{Prácticas filosóficas en la infancia}

Este resultado justifica la tendencia de las prácticas en la PFI y asume las transformaciones y posibilidades relacionadas con el uso de materiales, propuestas y textos que esbozan el encuentro con los ambientes de paz. Los discursos revelan que los referentes teóricos movilizan las discusiones y que los problemas abordados son un insumo fundamental para el trabajo en el aula. Así, Lipman $(1988,2016)$ y Pineda (2004) son referentes básicos en 
el ejercicio ético y político y en las prácticas desarrolladas. Las actitudes de los docentes reflejan la transformación de pensamiento y las miradas hacia la filosofía. Por ejemplo, confianza, tranquilidad, apertura, flexibilidad y autoformación contribuyen a fortalecer el pensamiento crítico y reflexivo, e incluso formas distintas de relacionamiento con el pensamiento representadas en las prácticas, efectos y transformación.

Prácticas. Un soporte teórico-metodológico es la línea de investigación que apoya el desarrollo, la asesoría y la organización de encuentros académicos, en donde se dialogan y se fortalecen las prácticas para la construcción de paz. En efecto, la vinculación entre la universidad y la sociedad se reconoce como un respaldo y un sustento que contribuye a la formación de redes. También convoca a la transformación de prácticas pedagógicas, e incluso de instituciones educativas: "pertenecer a la línea de investigación filosofía e infancia es el mismo trabajo de formación que yo he hecho. Es la misma transformación que ha sufrido el colegio" (E7). Las propuestas desarrolladas consolidan espacios de participación para docentes y estudiantes en torno al cuestionamiento y a la reflexión sobre los acontecimientos, experiencias y tensiones que la contemporaneidad trae a la escuela. Las prácticas promovidas en perspectiva filosófica determinan modos de proponer y de plantear alternativas en las escuelas. Por esto, tienen un lugar fundamental las actividades que promueven la formación autónoma con niños y niñas.

FpN y la PFI son cajas de herramientas, puesto que contienen elementos caracterizados como rizomáticos ${ }^{3}$ y se trasladan a diferentes espacios, momentos e intenciones. Por ejemplo, la comunidad de indagación como organización transforma relaciones y espacios de poder al haber un distanciamiento en las dinámicas tradicionales y en el lugar del profesor y del estudiante, para que "el chico pueda desde preguntas él mismo generar un conocimiento en donde pueda él pensarlo" (E6). Las habilidades comunicativas propician las interrelaciones y la construcción de acuerdos. Hay prácticas como las Olimpiadas Filosóficas, que son instrumentos que movilizan la comunicación. El reconocimiento de los niños como promotores de cambio se presenta a partir de que "los estudiantes hagan el ejercicio de razonar sobre su propio pensamiento" (E7). La formación autónoma se relaciona con la responsabilidad y con el cuidado. Una práctica que los materializa son los semilleros de investigación, donde "se empiezan a crear líderes en otros espacios, en otros aspectos" (E4).

Efectos. Los efectos se estructuran como las prospectivas y aquellas ideas que se pueden lograr a través de la implementación del programa. Las percepciones y las proyecciones identificadas son basadas en hechos y experiencias de los docentes en las escuelas. La FpN es el resultado de formular preguntas y consolidarlas en respuestas o contrapreguntas, en donde se trazan inicios, continuaciones, rupturas y finales. De este modo, los docentes exponen los efectos del programa relacionados con el pensar, con el lenguaje, con los procesos comunicativos y con la formación del ser.

El pensar como ejercicio es valorado en la reflexión y en la transformación de las formas de pensar en los niños. La actividad de razonar y el desarrollar habilidades de pensamiento configuran nuevos espacios, ambientes y situaciones, que adquieren sentido y significado para leer el contexto. Las prácticas de lecturas con el otro, narrarse a partir de la vivencia

3 En términos de Deleuze, lo rizomático de los elementos del trabajo en PFI se asume a través de las circunstancias, actos, organización que conforman puntos que conectan, interrumpen y rompen líneas, así que las prácticas son relacionadas con la articulación, segmentariedad, formación de territorialidades, líneas y movimientos de fuga (Deleuze \& Guattari, 1977). 
y escribirse en otros términos son productos de "reflexión y cambio de pensamiento. Nos lleva a dar buenas razones... [para que] nos den, nos aclaren, nos ayuden a la comunidad" (E5) a generar oportunidades permanentes de contribución a la escuela y a la sociedad, en un camino de la transformación.

La FpN es importante en el proceso de comunicación y de exploración de pensamientos en diferentes lenguajes: "es muy importante la palabra, el argumento y el discurso" (E2). Comunicar a través de la palabra, de lo escrito o del gesto reafirma que la expresión es fundamental para evocar la activación del pensamiento. Así, la interacción del lenguaje y el pensamiento articula decisión y actuación, posibilita la enunciación de conceptos e intensifica formas de leer, de asumir y de reflexionar la realidad. La palabra y el pensamiento ayudan a "construir la voz de un niño" (E4) en las comunidades de investigación.

Por último, la filosofía es una forma de vida (Hadot, 2009) y configura un ethos: "es una forma de ser y pensar las cosas de una forma distinta, donde lo primero que tú haces es el reconocimiento y la legitimidad del otro" (E7). La filosofía, en el encuentro con el otro, promueve una construcción del ser y garantiza el ejercicio de coexistencias y la constitución de maneras particulares de ser. En la consolidación de sujetos ético-políticos, la FpN asume que cada disposición, cada decisión y cada acción comparte, impacta e influye en la apreciación de sí mismo, de otro sujeto y del entorno.

Transformación. Las experiencias atraviesan al sujeto y le permiten su transformación. Por ello, se distinguen esferas de transformación en relación con el saber, con la institución y con el sujeto. Hay apreciaciones alrededor de la filosofía como asignatura. La lectura y la escritura son saberes que se movilizan en las escuelas. Los docentes manifiestan que existe mayor interés porque FpN despierta participación y gusto. Así mismo, la articulación, el análisis, la interpretación y la participación revelan la transformación de la comunicación y del pensamiento. Incluso, esta transformación se refleja en las interrelaciones con la comunidad, por ejemplo, en y con padres de familia. La implementación y las experiencias en la PFI transforman la forma de ser de los niños y las relaciones que establecen consigo y con los demás.

\section{Acciones y proyecciones para construir paz en la escuela}

Las maneras de construir paz en la escuela se enriquecen al consolidar relaciones basadas en principios ético-políticos. Las representaciones en comunidades escolares puntualizan en prevenir agresiones y conflictos, y en medidas de prevención ante el abuso.

Esta categoría reúne la construcción de paz referida a la resolución de conflictos, las condiciones para construir paz y los insumos para la paz.

Resolución de conflictos. La convivencia y la resolución de conflictos pacíficamente implican el reconocimiento de un tejido social que se consolida gracias a la formación de actitudes, de habilidades y de principios dialógicos. En estos términos, el fomento de la alteridad, las interrelaciones y las conexiones que se establecen entre una cultura son importantes y necesarias para responder éticamente ante dificultades. Dichas soluciones se plantean bajo principios de razonabilidad y de un conocimiento de un pasado como antecedentes que se configuran en maneras de decidir y de actuar.

Los discursos y las prácticas para resolver conflictos y construir ambientes éticos y 
políticos se configuran en comunidades reflexivas y conscientes de los pensamientos, de las palabras y de los hechos, al considerar el diálogo y la escucha y asumir los actos y la mediación como principios que posibilitan acuerdos, equilibrios y paz. Por ejemplo, está la institucionalización de las comisiones de ética, como "espacios en que los niños pudieran gestionar lo que les pasaba... manteniendo la estrategia, empoderando a los niños todos los periodos para que ejerzan la comisión de ética” (E7). Estas comisiones se concretan en los espacios escolares a través del alcance de acuerdos, la mediación entre estudiantes (ÁlvarezMaestre \& Pérez-Fuentes, 2019), el entendimiento del conflicto, la reflexión y la conciencia de lo sucedido. En esta experiencia se visibiliza la capacidad de razonar sobre las acciones y de responder acertadamente desde la ética. Así mismo, permite que los estudiantes representen una figura de conocimiento y razonabilidad, y que sean capaces de decidir y de tomar posturas objetivas ante una problemática.

Condiciones para construir paz. La construcción de paz se expresa en el cultivo de actitudes y en el desarrollo de propuestas que se generan en la escuela, pero que se proyectan fuera de ella. A nivel institucional, se pretende que en las aulas se comprometan tanto docentes como estudiantes a forjar una corresponsabilidad con la ciudadanía, que genere aperturas a ser críticos ante la realidad, a ser conscientes de lo que sucede y a manifestarse. Por esto, necesitan "espacios para pensar, espacios para decidir y espacios para que los niños puedan actuar en autonomía" (E2). La lectura y la comprensión de la realidad tienen lugar en los objetivos de formación y se alimentan con la participación de las familias, las cuales son el sustento para la continuación de procesos y acciones para favorecer la convivencia y la resolución de conflictos de manera pacífica, con apoyo en el "pensamiento lógico, el pensamiento estético, el histórico-social y ambiental" (E4).

La posición del sujeto y la construcción de los modos de subjetivación son una entrada a configurar territorios de paz: "los niños que aprendan que son un territorio de paz. Ellos como seres humanos no pueden venir a violentarse por ninguna persona" (E2). La construcción de paz invita a reconocer que los individuos son pensantes y críticos, y que las voces de infancia expresan, dialogan, preguntan y comparten su visión del mundo, donde la reflexión y la conciencia producen soluciones creativas y cuidadosas para la transformación de aquello que genera transgresión y violación.

Insumos para la paz. Experienciar el pensamiento es una entrada a la construcción de paz y a propiciar ambientes y escenarios de convivencia pacífica. La inserción de la filosofía y el potenciamiento del ejercicio del pensar configuran espacios y miradas para comprender acontecimientos que busquen el beneficio de las comunidades y de los estudiantes en la escuela. Esto implica identificar las habilidades que requieren mayor atención para su desarrollo y proponer ejercicios para su fortalecimiento. Por ello, el plano vivencial establece aperturas para indagar y decidir con autonomía y heteronomía. Así, la comprensión de la alteridad garantiza la preocupación por el otro y el cuidado que merece cada sujeto que convive en una sociedad. La alteridad posibilita espacios de diálogo y de discusión, como se refleja en las comunidades de indagación.

Otra entrada es la concepción de infancia, de niño y de sujeto como potencia y pensamiento, que genera efectos en la construcción de paz, en tanto que las narrativas, las expresiones, los lenguajes y las sensibilidades son partícipes de una realidad modificable. 
Así pues, la constitución de los sujetos cuestiona y propicia que los espacios se deconstruyan y respondan a las necesidades y expectativas que emergen de las condiciones de bienestar y de la preocupación por el lugar de la infancia.

Como última entrada, la comunidad de docentes permite que se compartan sentires y percepciones para nuevos intentos de hacer escuela, de modo que la voluntad, la motivación y la pasión inspiren actividades de transformación y la habitación de espacios y de territorios con sentidos y significados para niños, niñas y jóvenes; es decir, que se forje una escuela para la libertad. A su vez, se necesita el respaldo institucional de los centros educativos a todos los proyectos, propuestas y alternativas que se gestionen en las escuelas. Por lo tanto, se trata de fortalecer los vínculos y la institucionalización de acciones, para que se garanticen oportunidades y continuidades en propuestas para la construcción de paz (Giraldo et al., 2018).

Por último, la escuela como el lugar de institucionalización de saberes es el espacio propicio para fomentar la creatividad, el cuidado y la preocupación por el bienestar en comunidad, con miras a proyectar transformaciones en las relaciones humanas de respeto por la vida, por la dignidad y por los derechos fundamentales, para la construcción de paz como compromiso común.

\section{Conclusiones}

Los discursos de los profesores ubican la PFI como una forma de proponer maneras de construir la paz en escuelas rurales. Las prácticas representan un impulso para generar ambientes de convivencia alrededor del cuidado y del pensamiento. Así, el ejercicio de pensar es el que provoca el reconocimiento y la conciencia de preocuparse por la presencia de un otro. El cuidado se asume como un principio detonante de alteridad, de respeto y de toma de conciencia, que implica valorar y proteger a los sujetos con los que se comparte un espacio. Así mismo, las palabras y las actuaciones se leen en clave de efectos y consecuencias, en tanto se proyecte un razonamiento justificado y argumentado para decidir por las mejores maneras de proceder en un conflicto. También, se resalta que la formación de personas ético-políticas es una misión constante en búsqueda del cuidado de sí mismo, del otro y de lo otro. Esto se logra con el diálogo, puesto que los procesos comunicativos posibilitan que se active el pensamiento en comunidad, ya que la escucha, el silencio, la enunciación y la corporalidad descubren maneras de conducir los conflictos y de buscar las formas más pertinentes para resolver inconvenientes. Así mismo, la pregunta se ubica en un lugar de toma de conciencia, de empoderamiento y de fortalecimiento de la acción. Su cultivo permite que se generen espacios reflexivos y de resistencia importantes para la construcción de paz y de ambientes de convivencia en la realidad, no solo en la escuela, sino en el país.

\section{Referencias}

Agencia de Desarrollo Rural. (2019). Plan integral de desarrollo agropecuario y rural con enfoque territorial, departamento de Boyacá. https://www.adr.gov.co/servicios/pidaret/ BOYACA\%20TOMO\%201.pdf.

Álvarez-Maestre, A., \& Pérez-Fuentes, C. (2019). Educación para la paz: aproximación teórica desde los imaginarios de paz. Educación y Educadores, 22(2), 277-296. https:// 
doi.org/10.5294/edu.2019.22.2.6

Arboleda, Z., Herrera, M., \& Prada, M. (2017). ¿Qué es educar y formar para la paz y cómo hacerlo? Educación y Pedagogía para la Paz. Oficina del Alto Comisionado para la Paz; Acción CaPaz: Estrategia de Capacidades para la Paz y la Convivencia. https://educrea. cl/que-es-educar-y-formar-para-la-paz-y-como-hacerlo/

Congreso de Colombia. (2014, 1 de septiembre). Ley 1732 de 2014 por la cual se establece la Cátedra de la Paz en todas las instituciones educativas del país. https://www.mineducacion. gov.co/1759/w3-article-381604.html?_noredirect=1

Deleuze, G. \& Guattari, F. (1977). Rizoma. Introducción. Pre-textos.

Giraldo, M., Amorocho, E., Granados, J., Hilarión, M., \& Arias, R. (2018). Reflexiones de la educación para la paz en tiempos de postconflicto en Colombia. Revista de Estudios Socioeducativos, ReSed, (6), 118-128. https://revistas.uca.es/index.php/ReSed/article/ view/S.2.8N6

Gobierno Nacional, \& FARC-EP (2016). Acuerdo final para la terminación del conflicto y la construcción de una paz estable y duradera. https://www.jep.gov.co/Normativa/Paginas/ Acuerdo-Final.aspx

Hadot, P. (2009). La filosofía como forma de vida. Alpha Decay.

Hernández, C. (Coord.). (s. f.). Monografía político electoral. Departamento de Boyacá 19972007. Misión de Observación Electoral; Corporación Nuevo Arcoíris. https://moe.org. co/home/doc/moe_mre/CD/PDF/boyaca.pdf.

Larrosa, J. (1995). Escuela, poder y subjetivación. Ediciones de la Piqueta.

Lipman, M. (1988). Philosophy Goes to School. Temple University Press.

Lipman, M. (2016). El lugar del pensamiento en la educación. Octaedro.

Martínez-Restrepo, R., Pertuz, M., \& Ramírez, J. (2016). La situación de la educación rural en Colombia. Los desafíos del posconflicto y la transformación del campo. Compartir; Fedesarrollo. https://compartirpalabramaestra.org/documentos/fedesarrollo_compartir/ la-situacion-de-la-educacion-rural-en-colombia-los-desafios-del-posconflicto-y-latrasformacion-del-campo.pdf

MEN. (2015). Orientaciones generales para la implementación de la Cátedra de la Paz en los establecimientos educativos de preescolar, básica y media de Colombia. http://aprende. colombiaaprende.edu.co/ckfinder/userfiles/files/orientacionesedupaz.pdf

MEN. (2017). Plan especial de educación rural hacia el desarrollo rural y la construcción de paz. http://www.congresoeducacionruralcoreducar.com/images/Doc_web/10.PEER_06-2017.pdf.

Misión para la Transformación del Campo. (2015) El campo colombiano: un camino hacia el bienestar y la paz. Tomo 3. Departamento Nacional de Planeación. https://colaboracion. dnp.gov.co/CDT/Agriculturapecuarioforestal\%20y\%20pesca/TOMO\%203.pdf

Observatorio de Memoria y Conflicto \& Centro de Memoria Histórica. (2019). El conflicto armado en cifras. http://micrositios.centrodememoriahistorica.gov.co/observatorio/ 
portal-de-datos/el-conflicto-en-cifras/

Pagni, P. (2016). Ética da amizade e deficiência: outras formas de convívio com o devir deficiente na escola. Childhood \& Philosophy, 12(24), 343-370. https://www.redalyc.org/ articulo.oa?id=512055734007

Pineda, D. (2004). La educación moral desde la perspectiva de "Filosofía para niños". En D. Pineda, \& W. Kohan (Orgs.), Pensamiento, acción y sensibilidad: la mirada de "Filosofía para niños" (pp. 183-215). Beta

Presidencia de la República de Colombia. (2015, 25 de mayo). Decreto 1038 de 2015, por el cual se reglamenta la Cátedra de la Paz. https://www.mineducacion.gov.co/1759/w3article-382203.html?_noredirect=1.

Rivas, I. (2017). La escuela como escenario facilitador de paz: panorama de trabajos sobre una formación ética en escolares, para la construcción de mejores relaciones de convivencia dentro y fuera del aula. Revista Educación y Ciudad, (31), 107-117. https:// revistas.idep.edu.co/index.php/educacion-y-ciudad/article/view/1613

Torre de la, G., Carlo di, E., Florido, A., Opazo, H., Ramírez, C., Rodríguez, P., Sánchez, A., \& Tirado, J. (2013). Teoría Fundamentada o Grounded Theory. Universidad Autónoma de Madrid. http://mastor.cl/blog/wp-content/uploads/2016/01/Teoriafundamentada.Una-sintesis.-pdf.pdf.

Trivelli, C., \& Berdegué, J. (2019). 2030. Transformación rural:pensando el futuro de América Latina y el Caribe. Alimentación, agricultura y desarrollo rural en América Latina y el Caribe. Organización de las Naciones Unidas para la Alimentación y la AgriculturaFAO. http://www.fao.org/3/ca5508es/ca5508es.pdf.

Unesco. (2017). La filosofía, una escuela de la libertad. http://dcsh.izt.uam.mx/cen_doc/ cefilibe/images/libros-e/01-Filosofia-escuela-de-la-libertad.pdf

Unidad para la Atención y Reparación Integral a las Víctimas. (2020). Registro único de víctimas. Víctimas de conflicto armado. www.unidadvictimas.gov.co/es/ruv/37385. 